\title{
Characteristics of rapid vs slow progression to type 1 diabetes in multiple islet autoantibody-positive children
}

\author{
P. Achenbach • M. Hummel • L. Thümer • \\ H. Boerschmann • D. Höfelmann • A. G. Ziegler
}

Received: 29 September 2012 / Accepted: 6 March 2013 /Published online: 29 March 2013

(C) Springer-Verlag Berlin Heidelberg 2013

\begin{abstract}
Aims/hypothesis Islet autoantibody-positive children progress to type 1 diabetes at variable rates. In our study, we asked whether characteristic autoantibody and/or gene profiles could be defined for phenotypes showing extreme progression.

Methods Autoantibodies to insulin (IAA), GAD (GADA), insulinoma-associated antigen-2 (IA-2A) and zinc transporter 8 (ZnT8A) were measured in follow-up sera, and genotyping for type 1 diabetes susceptibility genes (HLA$D R / H L A-D Q$, INS variable number of tandem repeats [VNTR] and single nucleotide polymorphisms at PTPN22, PTPN2, ERBB3, IL2, SH2B3, CTLA4, IFIH1, KIAA0350 [also known as CLEC16A], CD25, IL18RAP, IL10, COBL) was performed on the DNA samples of children born to a parent with type 1 diabetes and prospectively followed from birth for up to 22 years.

Results Of the 1,650 children followed, 23 developed multiple autoantibodies and progressed to diabetes within 3 years (rapid progressors), while 24 children developed multiple autoantibodies and remained non-diabetic for more
\end{abstract}

P. Achenbach and M. Hummel contributed equally to this study.

Electronic supplementary material The online version of this article (doi:10.1007/s00125-013-2896-y) contains peer-reviewed but unedited supplementary material, which is available to authorised users.

P. Achenbach $(\bowtie) \cdot$ D. Höfelmann • A. G. Ziegler $(\bowtie)$ Institute of Diabetes Research, Helmholtz Zentrum München, Ingolstaedter Landstrasse 1,

85764 Neuherberg, Germany

e-mail: peter.achenbach@helmholtz-muenchen.de

e-mail: anette-g.ziegler@helmholtz-muenchen.de

P. Achenbach · L. Thümer · D. Höfelmann · A. G. Ziegler

Forschergruppe Diabetes, Klinikum rechts der Isar,

Technische Universität München, Munich, Germany

P. Achenbach • M. Hummel • H. Boerschmann • A. G. Ziegler

Forschergruppe Diabetes e.V, Neuherberg, Germany than 10 years from seroconversion (slow progressors). Rapid and slow progressors were similar with respect to $H L A$ $D R / H L A-D Q$ genotypes, development of IAA, GADA and $\mathrm{ZnT} 8 \mathrm{~A}$, and progression to multiple autoantibodies. In contrast, IA-2A development was considerably delayed in the slow progressors. Furthermore, both groups were effectively distinguished by the combined presence or absence of type 1 diabetes susceptibility alleles of non-HLA genes, most notably IL2, CD25, INS VNTR, IL18RAP, IL10, IFIH1 and $P T P N 22$, and discrimination was improved among children carrying high-risk $H L A-D R / H L A-D Q$ genotypes.

Conclusions/interpretation Our data suggest that genotypes of non-HLA type 1 diabetes susceptibility genes influence the likelihood or rate of diabetes progression among children with multiple islet autoantibodies.

Keywords Islet autoantibody · Progression · Susceptibility genes $\cdot$ Type 1 diabetes

$\begin{array}{ll}\text { Abbreviations } \\ \text { aa } & \text { Amino acid } \\ \text { AIC } & \text { Akaike's information criterion } \\ \text { GADA } & \text { Autoantibodies to GAD } \\ \text { IA-2A } & \text { Autoantibodies to insulinoma-associated antigen-2 } \\ \text { IAA } & \text { Autoantibodies to insulin } \\ \text { IQR } & \text { Interquartile range } \\ \text { SNP } & \text { Single nucleotide polymorphism } \\ \text { VNTR } & \text { Variable number of tandem repeats } \\ \text { ZnT8A } & \text { Autoantibodies to zinc transporter } 8\end{array}$

\section{Introduction}

Type 1 diabetes is preceded by a preclinical period of autoimmunity directed against the pancreatic islet beta cells [1]. This islet autoimmunity can develop early in life, and its initiation is signalled by the seroconversion of autoantibodies to insulin 
(IAA), GAD (GADA), insulinoma-associated antigen-2 (IA-2A) and/or zinc transporter 8 (ZnT8A) [2]. Islet autoimmunity and type 1 diabetes affect an increasing number of people worldwide, the strongest increase in incidence being seen in young children [3]. The rise in incidence is clearly environmentally induced, and it is likely that environmental factors not only contribute to the higher number of people with autoimmune diabetes, but also interact with type 1 diabetes susceptibility genes to affect the rate of progression to disease. The rate of progression from islet autoantibody seroconversion to type 1 diabetes varies considerably between individuals, ranging from a few months to many years [4-6], and recent data from Germany suggest that it has been accelerating over the last 20 years [7].

Earlier studies have shown that one key step in disease pathogenesis and determination of the risk of progression is spreading of the autoantibody response to multiple islet autoantigens [8,9], but multiple vs single islet autoantibody positivity cannot completely explain the variation seen in the rate of development of type 1 diabetes. We believe that it is important to understand the mechanisms regulating the progression from autoimmunity to clinical diabetes, and to identify signatures and biomarkers that allow individuals to be classified into rapid or slow progressor phenotypes. The aim of our study was therefore to perform a detailed characterisation and comparison of children who rapidly progress to diabetes within 3 years of islet autoantibody seroconversion or have a long diabetes-free survival of at least 10 years after seroconversion. We took advantage of information on children with multiple autoantibodies from the prospective BABYDIAB cohort study, in which we have followed children with a family history of type 1 diabetes from birth for over 20 years [10].

\section{Methods}

Study cohort, participants and samples The study was performed on children from the BABYDIAB study, a longitudinal study examining the natural history of islet autoimmunity and type 1 diabetes in 1,650 children born to a mother or a father with type 1 diabetes [4]. Recruitment began in July 1989 and ended in February 2001. All the children were recruited from Germany. The cohort was not population based, and $97 \%$ of families were white German. Venous blood samples were obtained from the children at study visits scheduled at 9 months and at 2, 5, 8, 11, 14, 17 and 20 years of age.

Islet autoantibodies (IAA, GADA, IA-2A and ZnT8A) were measured in samples taken at all the scheduled visits. If children had a positive autoantibody finding, their families were asked to provide a sample for confirmation of autoantibody status within 6 months and provide samples yearly.
Samples were no longer collected if a child developed diabetes. Families were asked to report the occurrence of symptoms of diabetes. In children with islet autoantibodies, a yearly OGTT was performed. Onset of diabetes was defined according to ADA criteria [11]. The cumulative dropout rate in BABYDIAB was $2 \%, 8 \%, 17 \%$ and $23 \%$ by age $2,5,8$ and 11 years, respectively. The median follow-up time from birth to the last sample was 12.5 years (interquartile range [IQR] 6.5-15.5 years) and from birth to diabetes or last contact was 14.1 years (IQR 11.9-15.6 years).

The BABYDIAB study was approved by the ethical committee of Bavaria, Germany (Bayerische Landesärztekammer No. 95357). All families gave written informed consent to participate in the study. Investigations were carried out in accordance with the principles of the Declaration of Helsinki, as revised in 2000 .

Islet autoantibody measurements IAA, GADA, IA-2A, and ZnT8A were determined using radiobinding assays as previously described $[2,10]$. Briefly, IAA was measured by protein $\mathrm{A} / \mathrm{G}$ radiobinding assays using $\left[{ }^{125} \mathrm{I}\right]$-recombinant human insulin labelled at tyrosine amino acid (aa)14. GADA, IA-2A and ZnT8A were measured by protein A radiobinding assays using $\left[{ }^{35} \mathrm{~S}\right]$-methionine-labelled in vitro transcribed/translated recombinant human GAD65 (aa1aa585), the intracellular portion of insulinoma-associated antigen-2 (aa605-aa979) and COOH-terminal (aa268aa369) constructs of the zinc transporter 8 R325 and W325 variants, respectively. Samples were considered to be ZnT8A positive if autoantibodies to at least one of the zinc transporter 8 variants (ZnT8RA and/or ZnT8WA) were found. The upper limit of normal for each assay was determined using Q-Q plots and corresponded to the 99th percentile of 836 control children. Children were considered islet autoantibody positive when two consecutive serum samples were positive.

Autoantibody assays were evaluated by the Diabetes Antibody Standardization Program, and the performances are shown as those of laboratory 121 in published reports [12-14]. The assays had sensitivities and specificities of $86 \%$ and $93 \%$ (GADA) [12], $72 \%$ and $100 \%$ (IA-2A) [12], $70 \%$ and $99 \%$ (IAA) [13], $68 \%$ and $100 \%$ (ZnT8RA) [14] and $51 \%$ and $100 \%$ (ZnT8WA) [14] in proficiency workshops. The interassay CVs for samples with a low autoantibody titre were 18\% (GADA), 16\% (IA-2A), 11\% (IAA), 17\% (ZnT8RA) and 16\% (ZnT8WA), respectively.

Genotyping HLA class II alleles HLA-DRB1, HLA-DQA1 and $H L A-D Q B 1$ were determined using PCR-amplified DNA and non-radioactive sequence-specific oligonucleotide probes, and $H L A-D R / H L A-D Q$ genotypes were categorised according to risk of type 1 diabetes into high risk, moderate risk, neutral and protective genotypes, as 
previously described [15] (see electronic supplementary material [ESM] Table 1). INS variable number of tandem repeats (VNTR) alleles were determined using HphI digestion of PCR amplification products of the region of interest, as previously described [15]. An additional 12 type 1 diabetes susceptibility gene single nucleotide polymorphisms (SNPs) were selected for typing on the basis of the genes that were reported to be susceptible in 2009 [16], the strength of their OR and the SNP-typing that has successfully been established in Munich: PTPN22 rs6679677, PTPN2 rs1893217, ERBB3 rs2292239, IL2 rs4505848, SH2B3 rs3184504, CTLA4 rs3087243, IFIH1 rs2111485, KIAA0350 (also known as CLEC16A) rs12935413, CD25 rs11594656, IL18RAP rs917997, IL10 rs3024505, and COBL rs4948088. Genotyping of these SNPs was performed with the MassARRAY system using iPLEX chemistry (Sequenom, San Diego, CA, USA), as previously described [17]. To control for reproducibility, $16 \%$ of samples were genotyped in duplicate with a discordance rate $<0.5 \%$. All SNPs were tested for deviation from Hardy-Weinberg equilibrium by means of a $\chi^{2}$ or Fisher's exact test.

Statistical analysis Prevalence was compared between groups using Fisher's exact test. The Mann-Whitney $U$ test was used to compare continuous variables between groups. The cumulative frequency for each of IAA, GADA, IA-2A and ZnT8A, and respectively for any of the four autoantibodies, was calculated using life-table analysis. A child was considered to have a positive autoantibody outcome if at least two consecutive samples were found to be positive for the autoantibody. Positive results that were likely to be due to a placental transfer of maternal autoantibodies were excluded. The age at the first autoantibody-positive sample was used as the time to event. Analysis considered censoring for losses to follow-up. In order to calculate the risk of progression to multiple autoantibodies, the time between the age at first appearance of any of the four autoantibodies (the seroconversion age) and the age at appearance of two or more of IAA, GADA, IA-2A and/or ZnT8A was used as the time to event. The significance of the differences between groups was determined using the logrank test.

Risk scores were generated for non-HLA genotypes as previously described [17]. For each gene, a score of 2 was given if the child was homozygous for the allele predisposing to type 1 diabetes, 1 if the child was heterozygous and 0 if the child was homozygous for the nonsusceptible allele. The sum of the scores was assigned as the combined risk score for each child. Combined risk scores were generated for permutations of multiple nonHLA genes $\left(n=2^{a}-1\right.$, where $a$ is the number of genes in the analysis) as previously described [17] and compared between groups using the Mann-Whitney $U$ test and twosample permutation test [18].
In order to assess the difference in discriminatory strength between alternative models derived from the combined risk scores, we used Akaike's information criterion (AIC) [19]. The AIC is based on the negative base $e \log$ likelihood $\left(\log _{e} L\right)$ and the number of variables $(k)$ of the model, and is defined as: $A I C=-2 \log _{e} L+2 k$. The lower the AIC, the better the model. $\log L$ was determined by logistic regression analysis using the combined risk scores as the continuous covariate, and rapid or slow progression as the binary dependent covariate in each model. For all analyses, two-tailed $p$ values were used and considered significant at $p<0.05$ or $p<0.05 / k$ in case of multiple comparisons (Bonferroni's correction). The statistical analyses were performed using GraphPad Prism 3 (GraphPad Software, San Diego, CA, USA), the Statistical Package for the Social Sciences (SPSS 19.0; SPSS, Chicago, IL, USA), MATLAB software (R2011a; MathWorks, Nattick, MA, USA) and statistical software R 2.14.1 (http://cran.r-project.org/web/ packages/perm).

\section{Results}

Identification of rapid and slow progressors By April 2012, a total of 156 children from the BABYDIAB cohort $(n=1,650)$ had developed IAA, GADA, IA-2A and/or ZnT8A with positive results in at least two consecutive blood samples, and had been followed from seroconversion for a median of 7.0 years (IQR 3.7-10.4 years). Eighty-one (52\%) of the 156 children had developed two or more of the islet autoantibodies, and 55 (35\%) had developed diabetes (Fig. 1).

In order to investigate the extreme progression phenotypes among the islet autoantibody-positive children, we classified children as (1) rapid progressors if they developed multiple autoantibodies and progressed to type 1 diabetes within 3 years after seroconversion, and (2) slow progressors if they developed multiple autoantibodies and remained non-diabetic for at least 10 years after seroconversion. Twenty-three children ( $15 \%$ of the islet autoantibodypositive BABYDIAB children) met the criteria of rapid progressors, and 24 children (15\%) the criteria of slow progressors (Fig. 1). Both groups seroconverted to autoantibody positivity at a young age (median 2.0 years [IQR 0.9 4.3] for rapid progressors vs 2.3 years [IQR 1.0-5.0] for slow progressors) (ESM Fig. 1a). A further 23 children developed type 1 diabetes after 3-10 years of follow-up from seroconversion (Fig. 1). These were classified as intermediate progressors and used as a confirmatory group in our study.

Characteristics of the autoantibody responses in rapid and slow progressors There was a high degree of similarity in 
Fig. 1 Selection of children with an extreme progression phenotype. The schematic diagram shows the number of children from the BABYDIAB study with respect to the development and status of islet autoantibodies $(\mathrm{AB})$, number of years of follow-up from seroconversion and progression to type 1 diabetes (T1D). Twenty-three children were classified as rapid progressors and 24 as slow progressors, and these were selected for further analysis

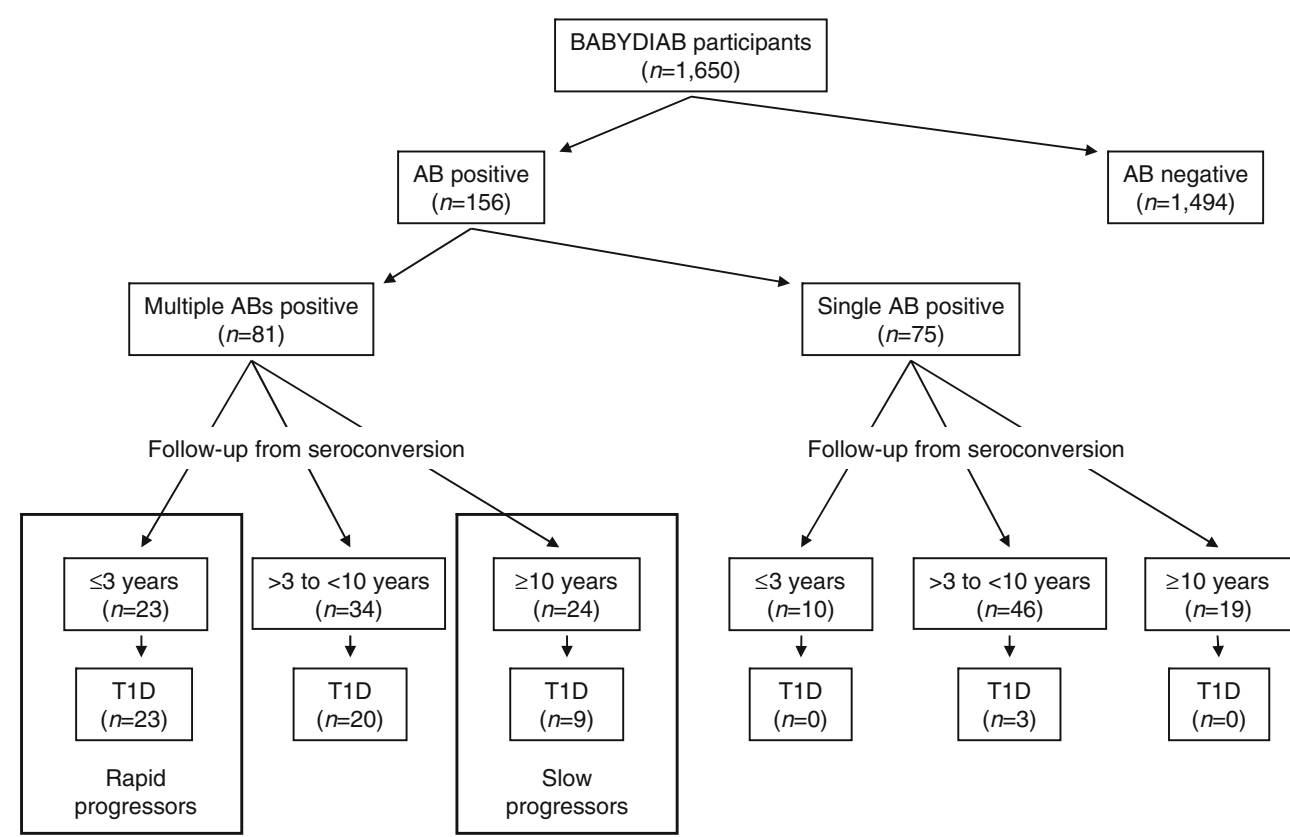

the islet autoantibody profiles of the rapid and slow progressors. No significant differences were observed with respect to the overall prevalence of IAA ( $91 \%$ in rapid vs $92 \%$ in slow progressors), GADA ( $87 \%$ vs $88 \%$ ), IA-2A (74\% vs $63 \%$ ) and ZnT8A ( $86 \%$ vs $83 \%$ ), as well as in terms of the maximal number of islet autoantibodies developed at any stage of follow-up (median 4 [IQR 3-4] vs 3 [IQR 3-4]). The rate of development of IAA, GADA and ZnT8A (Fig. 2a-c) and the rate of progression from single to multiple autoantibodies (ESM Fig. 1b) was also similar. However, one striking difference in the autoantibody profiles of the rapid and slow progressors was a considerably delayed development of IA-2A in the slow progressors $(p=0.002)$ (Fig. 2d). Among IA-2A-positive children, 16 (94\%) rapid progressors developed IA-2A within 18 months of autoantibody seroconversion, in contrast to only five (33\%) slow progressors $(p=0.0005)$.

Demographic and environmental factors Rapid and slow progressors were similar with respect to sex distribution (male, $57 \%$ vs $67 \% ; p=0.6$ ) and maternal type 1 diabetes status (diabetic mother, $65 \%$ vs $46 \% ; p=0.2$ ). Of note, 11 $(48 \%)$ of the 23 rapid progressors were delivered by Caesarean section, compared with only four $(17 \%)$ of the 24 slow progressors $(p=0.03)$.

Genetic characteristics of rapid and slow progressors HLA$D R / H L A-D Q$ risk categories were similarly distributed among rapid and slow progressors. Eleven children from each group carried high-risk $H L A-D R / H L A-D Q$ genotypes. We therefore asked whether non-HLA genotypes could aid in discrimination between the two groups. Complete a

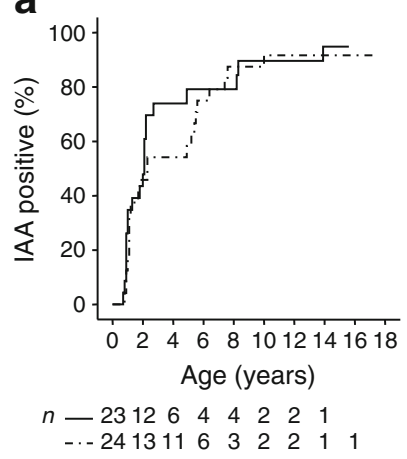

C

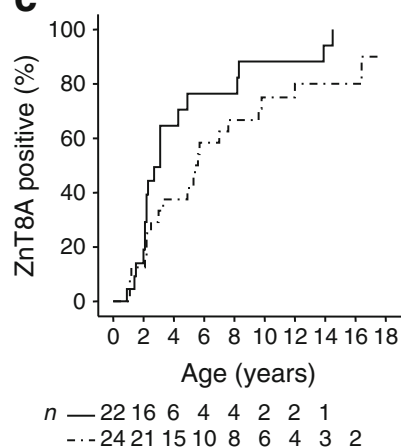

b

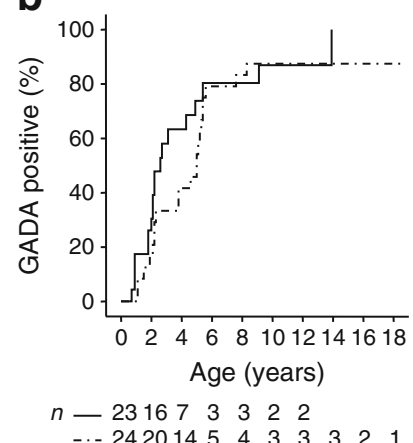

d

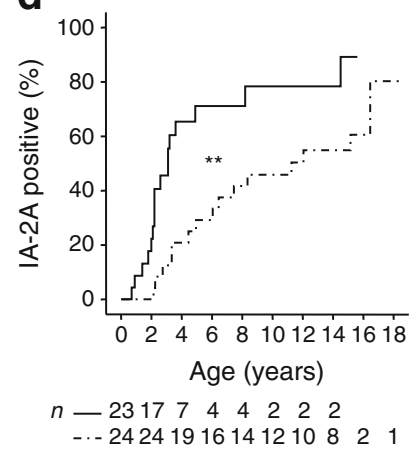

Fig. 2 Appearance of autoantibodies to biochemically defined target antigens. The cumulative life-table risk is shown for the development of (a) IAA, (b) GADA, (c) ZnT8A and (d) IA-2A among children classified as rapid progressors (solid line) or slow progressors (dotted line) in the BABYDIAB study. Slow progressors developed IAA, GADA and ZnT8A at a similar age to rapid progressors, but IA-2A at an older age $(p=0.002)$. Numbers below the $\mathrm{x}$-axis indicate the number of children in each group who were negative for the respective autoantibody and continued to be followed. $* * p<0.01$ 
successful typing for INS VNTR as well as SNPs of the 12 additional type 1 diabetes susceptibility genes was obtained for all 24 slow progressors and 19 of the 23 rapid progressors (i.e. DNA samples were not available for four children).

We first compared the prevalence of the allele predisposing to type 1 diabetes for each of the 13 non-HLA genes and revealed relatively minor differences between the two groups (ESM Table 2). Rapid progressors more frequently carried the predisposing allele of IL2 rs 4505848 ( $42 \%$ vs $15 \%$ among slow progressors; $p=0.007)$ and $I F I H 1$ rs $2111485(84 \%$ vs $65 \% ; p=0.05$ ) (ESM Table 2), and among children with highrisk $H L A-D R / H L A-D Q$ genotypes, the predisposing allele of CD25 rs 11594656 (95\% vs 64\%; $p=0.02$ ), IL10 rs3024505 $(90 \%$ vs $55 \% ; p=0.02)$ and $I F I H 1 \mathrm{rs} 2111485(85 \%$ vs $50 \%$; $p=0.02$ ) (ESM Fig. 2). However, none of these differences reached the level of significance after Bonferroni's correction.

We then analysed the combination of all non-HLA genotypes and generated combined risk scores for each child. The distribution of these risk scores was slightly, but significantly, different between rapid progressors (median 16, IQR 15-18) and slow progressors (median 15, IQR 14-16; $p=0.03$ ) (ESM Fig. 3). In order to further improve discrimination, we subsequently generated combined risk scores for all possible permutations of 12 non-HLA genes considering any number of genes in the permutation $\left(n=2^{12}-1\right.$, resulting in 4,095 gene combinations; level of significance after Bonferroni's correction, $p<1.2 \times 10^{-5}$ ), and compared the risk scores of the two groups using Mann-Whitney $U$ tests (range of $p$ values, 1.0 to $1.6 \times 10^{-5}$ ). COBL rs 4948088 was not included in this analysis because all but two children carried two risk alleles for this SNP (ESM Fig. 3). Twenty $(0.5 \%)$ of the 4,095 gene combinations yielded risk scores that discriminated between rapid and slow progressors with $p<10^{-4}$ (ESM Fig. 4).

The AIC of these 20 models ranged from 43.3 to 36.5 , compared with an AIC of 54.3 for our initial model that included all of the non-HLA genotypes, suggesting an overall improvement in discrimination between the rapid and slow progressors (ESM Fig. 4). The top two models with the lowest $p$ values and lowest AIC (model 1, $p=1.6 \times 10^{-5}$, AIC 36.5 ; model $2, p=1.7 \times 10^{-5}$, AIC 37.5 ) significantly discriminated between the two groups in the two-sample permutation test $\left(p=3.8 \times 10^{-6}\right.$ and $p=5.0 \times 10^{-6}$, respectively). These models were derived from risk scores that involved seven non-HLA genotypes, namely IL2 rs4505848, CD25 rs11594656, INS VNTR, IL18RAP rs917997, IL10 rs3024505, IFIH1 rs2111485, and PTPN22 rs6679677. Model 2 required the combination of all seven genotypes, whereas model 1 , which discriminated best between the rapid and slow progressors (Fig. 3), did not include the PTPN22 rs6679677 genotype. Both models discriminated particularly well between children carrying high-risk $H L A$ $D R / H L A-D Q$ genotypes (ESM Fig. 5).

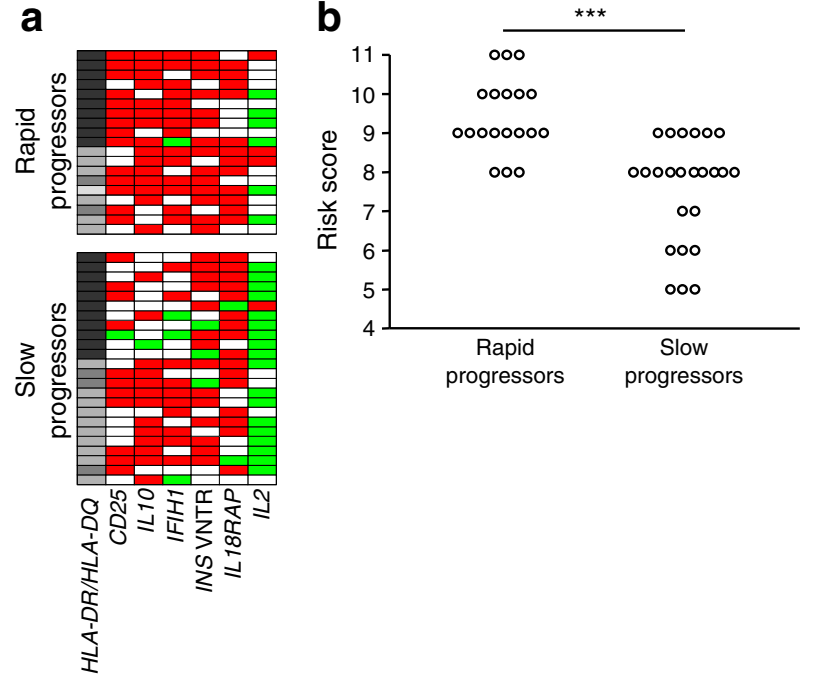

Fig. 3 The combination of genotypes of non-HLA type 1 diabetes susceptibility genes that discriminated best between rapid progressors $(n=19)$ and slow progressors $(n=24)$ in the BABYDIAB study. (a) HLA-DR/HLA-DQ, CD25 rs11594656, IL10 rs3024505, IFIH1 rs2111485, INS VNTR, IL18RAP rs917997 and IL2 rs4505848 genotypes are shown for each child. Non-HLA genes are ordered from left to right according to decreasing prevalence of the allele predisposing to type 1 diabetes among rapid progressors. $H L A-D R / H L A-D Q$ genotypes are categorised according to risk of type 1 diabetes into high risk, moderate risk, neutral and protective genotypes, and are colour-coded from dark grey (high risk) to light grey (protective). Non-HLA genotypes are also colour-coded and a score is given to each genotype, such that homozygosity for the predisposing allele (red) equals a score of 2 , heterozygosity (white) equals a score of 1 , and homozygosity for the non-susceptible allele (green) equals a score of 0 . Within each group of rapid and slow progressors, respectively, children are ordered from the top to the bottom row according to the presence or absence of high-risk $H L A-D R / H L A-D Q$ genotypes, and within these groups according to decreasing combined risk scores, which were calculated for each child as the sum of the genotype scores. (b) Combined risk scores are plotted for each child and compared between rapid and slow progressors $(p=0.000016) .{ }^{* * *} p<0.001$

In order to confirm our findings, we applied the two models to 21 children from the intermediate progressors group who were successfully genotyped for the respective non-HLA genes. Intermediate progressors had lower risk scores compared with rapid progressors (model $1, p=0.001$; model 2, $p=0.0004$ ), and by trend higher risk scores compared with slow progressors (Fig. 4), suggesting an inverse relationship between the risk scores and the time of progression.

\section{Discussion}

Identifying the characteristic signatures of progression in islet autoantibody-positive children may help to improve our understanding of the pathophysiological mechanisms underlying type 1 diabetes, so that effective prevention strategies can be developed and the most appropriate participants selected for clinical trials. We examined two groups 

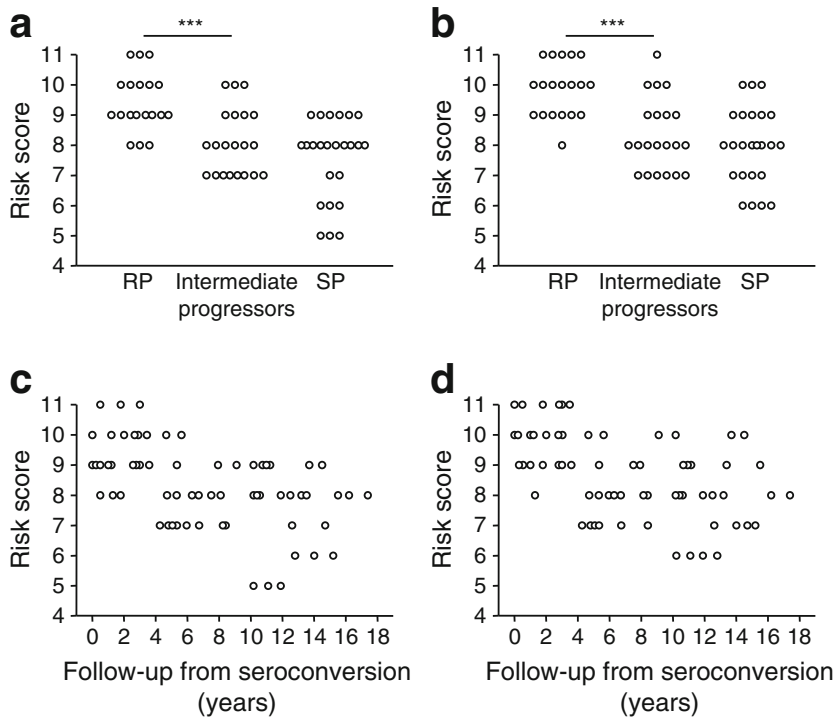

Fig. 4 (a, b) Risk scores of (a) model 1 and (b) model 2 are plotted for the group of rapid progressors (RP), slow progressors (SP) and children who developed type 1 diabetes after 3-10 years of follow-up from seroconversion (intermediate progressors), respectively. Both models distinguished between rapid and intermediate progressors (model 1, $p=0.001$; model 2, $p=0.0004)$. (c, d) Risk scores of (c) model 1 and (d) model 2 are plotted for the same children as in (a) and (b) with respect to the time of progression from islet autoantibody seroconversion. $* * * p<0.001$

of children who both developed an intense multiple islet autoantibody response but showed extremely different progression phenotypes, namely rapid progressors, who progressed to type 1 diabetes within 3 years of islet autoantibody seroconversion, and slow progressors, who remained diabetes-free for at least 10 years after seroconversion. We showed that rapid progressors were distinguished from slow progressors by a considerably faster development of IA-2A, an almost threefold higher frequency of delivery by Caesarean section, and an overall higher genetic load of alleles predisposing to type 1 diabetes from among the non-HLA susceptibility genes. Our study was made possible by the long follow-up of children with a family history of firstdegree relatives with type 1 diabetes in the BABYDIAB study [10], which enabled us to identify and study children with a phenotype of extreme progression.

The limitations of our study include the relatively small number of rapid and slow progressors, each comprising only $15 \%$ of the persistent islet autoantibody-positive children in BABYDIAB, the restricted number of type 1 diabetes susceptibility genes that were typed and analysed in our study, and the absence of a confirmatory cohort. We were also unable to assess the metabolic risk scores that were recently reported in autoantibody-positive participants of the Diabetes Prevention Trial-Type 1 [20]. It will therefore be important to test our findings in other cohorts, especially cohorts that are not restricted to children with a family history of type 1 diabetes.
An important finding was the high degree of similarity between the rapid and slow progressors with respect to the number and profile of islet autoantibodies, which are currently the best established markers for selecting participants for secondary prevention trials [21]. It has long been recognised that multiple autoantibody-positive individuals have a high risk of type 1 diabetes $[8,9,22]$, which can be further stratified by antibody characteristics such as target specificity $[23,24]$, titre $[24,25]$ or age at autoantibody seroconversion [4]. In particular, IA-2A has been reported as an immune marker associated with a very high risk of diabetes $[23,24]$. It is remarkable, therefore, that the only difference between the autoantibody responses in the rapid and slow progressors in our study was related to IA-2A. Rapid progressors developed IA-2A very early in the autoimmune process. The IA-2A frequency was similar in both groups, but IA-2A positivity was seen significantly later after initial autoantibody seroconversion in the slow progressors. This is consistent with IA-2A becoming a marker close to the onset of type 1 diabetes and heralding a more aggressive stage in the pathogenesis of the disease. Further antibody characteristics such as affinity and epitope specificity have been investigated in the BABYDIAB study $[2,26-28]$ and were highly consistent among multiple autoantibody-positive children.

More pronounced differences were seen with respect to the genetic background of the rapid and slow progressors. Notably, HLA-DR/HLA-DQ genotypes could not distinguish between rapid and slow progressors. In contrast, combinations of genotypes of IL2, CD25, IL10, IFIH1, INS VNTR, IL18RAP and PTPN22 were discriminative. Moreover, children with rapid and slow progression who carried high-risk HLA-DR/HLA-DQ genotypes could be discriminated by non-HLA genotype combinations.

The observed genetic associations are particularly intriguing with respect to the pathogenetic mechanisms underlying type 1 diabetes that have been proposed for causal allelic variants of the non-HLA susceptibility genes [29, 30]. It has been hypothesised that subsets of genes are acting to compromise pathways in type 1 diabetes that are related to IL-2 production and signalling [31-33]. Interestingly, 75\% of the slow progressors in our study, in contrast to only $26 \%$ of the rapid progressors, were homozygous for the nonsusceptible IL2 allele. In non-obese diabetic mice, homozygosity of the type 1 diabetes resistance allele of Il2 (Idd3) provides over $70 \%$ protection against diabetes [34]. Protective Idd 3 alleles translate into higher amounts of IL-2 production by autoreactive $\mathrm{T}$ cells and an enhanced development and function of T regulatory cells [32, 34, 35]. It is also noteworthy that, in human type 1 diabetes, the $I L 2$ gene was recently reported to be associated with the age of onset of diabetes [36]. Alleles of the $C D 25$ gene predisposing to type 1 diabetes lower the expression of the high-affinity alpha-chain (CD25) 
of the IL-2 receptor on the surface of the T cells, which affects IL-2 signalling and may result in an impaired function of the T regulatory cells $[31,33]$.

In addition to $I L 2$ and $C D 25$, several other loci associated with human type 1 diabetes encode molecules that play a role in the immune regulation of $\mathrm{T}$ cell-mediated adaptive immune responses, or of inflammatory signals provided by the innate immune system [30]. These include $I L-10$ [16, 37], IFIH1 [38, 39], IL18RAP [40] and PTPN22 [41], which were able to stratify progression in our study. Although a number of type 1 diabetes-associated gene SNPs did not significantly add to the discrimination between the rapid and slow progressors (i.e. COBL rs4948088, KIAA0350 rs 12935413 , CTLA4 rs3087243, SH2B3 rs3184504, ERBB3 rs2292239 and PTPN2 rs1893217), it is understood that susceptibility to type 1 diabetes, as well the progression rate from islet autoimmunity to clinical type 1 diabetes, is modified by other genes (reviewed in $[29,30])$ that have not been typed in our study. For example, we did not consider the effects of the HLA class I molecules, HLA-A and HLA-B [42], which present peptides to cytotoxic $\mathrm{CD} 8+\mathrm{T}$ cells and are strongly expressed in the pancreatic insulin-producing beta cells [43], or variants of the FUT2 gene that link type 1 diabetes susceptibility and resistance to infection [44]. Furthermore, our study did not consider the role of epigenetic changes, which may provide a molecular bridge between genes and environment in type 1 diabetes [30, 45].

In conclusion, our data suggest that differences in progression to clinical diabetes in children with multiple islet autoantibodies could largely be explained by the presence or absence of distinct non-HLA susceptibility alleles. Our findings may have consequences for future prevention studies for type 1 diabetes, namely in terms of strategies to select rapid progressors for and/or exclude slow progressors from respective clinical trials.

Acknowledgements The authors thank S. Krause, A. Gavrisan, (Forschergruppe Diabetes, Klinikum rechts der Isar, Technische Universität München, Germany), C. Matzke, M. Scholz, C. Kayser, E. Storz (Forschergruppe Diabetes e.V., Neuherberg, Germany), A. Knopff and C. Winkler (Institute of Diabetes Research, Helmholtz Zentrum München, Germany) for data collection and expert technical assistance; M. Hagen (Research Unit Scientific Computing, Helmholtz Zentrum München), H. Grallert (Research Unit Molecular Epidemiology, Helmholtz Zentrum München) and A. Beyerlein (Institute of Diabetes Research, Helmholtz Zentrum München) for analytical assistance; E. Bonifacio (Center for Regenerative Therapies, Dresden University of Technology, Germany) for scientific advice; and $\mathrm{M}$. Harsunen, E. Giannopoulou, and M. Krasmann (Forschergruppe Diabetes, Klinikum rechts der Isar, Technische Universität München) for clinical assistance. We also thank all paediatricians and family doctors in Germany for participating in the BABYDIAB study.

Funding This study was supported in part by grants from the German Federal Ministry of Education and Research (BMBF) to the Competence Network for Diabetes mellitus (FKZ 01GI0805) and the German Center for Diabetes Research (DZD e.V.), and from the Juvenile Diabetes Research Foundation (JDRF 17-2012-16).
Duality of interest The authors declare that there is no duality of interest associated with this manuscript.

Contribution statement PA analysed and interpreted the data and wrote the manuscript. AGZ is the principal investigator of the BABYDIAB study. MH and AGZ designed the study and contributed to data analysis and interpretation, and writing of the manuscript. LT, $\mathrm{HB}$, and DH contributed to data acquisition and analysis, and critically revised the manuscript for important intellectual content. All authors reviewed and approved the final manuscript.

\section{References}

1. Atkinson MA, Eisenbarth GS (2001) Type 1 diabetes: new perspectives on disease pathogenesis and treatment. Lancet 358:221-229

2. Achenbach P, Lampasona V, Landherr U et al (2009) Autoantibodies to zinc transporter 8 and SLC30A8 genotype stratify type 1 diabetes risk. Diabetologia 52:1881-1888

3. Patterson CC, Dahlquist GG, Gyurus E, Green A, Soltesz G, EURODIAB Study Group (2009) Incidence trends for childhood type 1 diabetes in Europe during 1989-2003 and predicted new cases 2005-20: a multicentre prospective registration study. Lancet 373:2027-2033

4. Hummel M, Bonifacio E, Schmid S, Walter M, Knopff A, Ziegler AG (2004) Brief communication: early appearance of islet autoantibodies predicts childhood type 1 diabetes in offspring of diabetic parents. Ann Intern Med 140:882-886

5. Steck AK, Johnson K, Barriga KJ et al (2011) Age of islet autoantibody appearance and mean levels of insulin, but not GAD or IA-2 autoantibodies, predict age of diagnosis of type 1 diabetes: diabetes autoimmunity study in the young. Diabetes Care 34:1397-1399

6. Parikka V, Nanto-Salonen K, Saarinen M et al (2012) Early seroconversion and rapidly increasing autoantibody concentrations predict prepubertal manifestation of type 1 diabetes in children at genetic risk. Diabetologia 55:1926-1936

7. Ziegler AG, Pflueger M, Winkler C et al (2011) Accelerated progression from islet autoimmunity to diabetes is causing the escalating incidence of type 1 diabetes in young children. $J$ Autoimmun 37:3-7

8. Bingley PJ, Christie MR, Bonifacio E et al (1994) Combined analysis of autoantibodies improves prediction of IDDM in islet cell antibody-positive relatives. Diabetes 43:1304-1310

9. Verge CF, Gianani R, Kawasaki E et al (1996) Prediction of type I diabetes in first-degree relatives using a combination of insulin, GAD, and ICA512bdc/IA-2 autoantibodies. Diabetes 45:926-933

10. Ziegler AG, Hummel M, Schenker M, Bonifacio E (1999) Autoantibody appearance and risk for development of childhood diabetes in offspring of parents with type 1 diabetes: the 2-year analysis of the German BABYDIAB Study. Diabetes 48:460-468

11. Puavilai G, Chanprasertyotin S, Sriphrapradaeng A (1999) Diagnostic criteria for diabetes mellitus and other categories of glucose intolerance: 1997 criteria by the Expert Committee on the Diagnosis and Classification of Diabetes Mellitus (ADA), 1998 WHO consultation criteria, and 1985 WHO criteria. World Health Organization. Diabetes Res Clin Pract 44:21-26

12. Torn C, Mueller PW, Schlosser M, Bonifacio E, Bingley PJ, Laboratories P (2008) Diabetes Antibody Standardization Program: evaluation of assays for autoantibodies to glutamic acid decarboxylase and islet antigen-2. Diabetologia 51:846-852

13. Schlosser M, Mueller PW, Torn C, Bonifacio E, Bingley PJ, Laboratories P (2010) Diabetes Antibody Standardization Program: evaluation of assays for insulin autoantibodies. Diabetologia 53:2611-2620 
14. Lampasona V, Schlosser M, Mueller PW et al (2011) Diabetes antibody standardization program: first proficiency evaluation of assays for autoantibodies to zinc transporter 8. Clin Chem 57:1693-1702

15. Walter M, Albert E, Conrad M et al (2003) IDDM2/insulin VNTR modifies risk conferred by IDDM1/HLA for development of type 1 diabetes and associated autoimmunity. Diabetologia 46:712-720

16. Barrett JC, Clayton DG, Concannon P et al (2009) Genome-wide association study and meta-analysis find that over 40 loci affect risk of type 1 diabetes. Nat Genet 41:703-707

17. Winkler C, Krumsiek J, Lempainen J et al (2012) A strategy for combining minor genetic susceptibility genes to improve prediction of disease in type 1 diabetes. Genes Immun 13:549-555

18. Agresti A, Mehta CR, Patel NR (1990) Exact inference for contingency tables with ordered categories. JASA 85:453-458

19. Akaike H (1974) A new look at the statistical model identification. IEEE Trans Autom Control 19:716-723

20. Sosenko JM, Krischer JP, Palmer JP et al (2008) A risk score for type 1 diabetes derived from autoantibody-positive participants in the Diabetes Prevention Trial-Type 1. Diabetes Care 31:528-533

21. Bingley PJ (2010) Clinical applications of diabetes antibody testing. J Clin Endocrinol Metab 95:25-33

22. Kulmala P, Savola K, Petersen JS et al (1998) Prediction of insulindependent diabetes mellitus in siblings of children with diabetes. A population-based study. The Childhood Diabetes in Finland Study Group. J Clin Invest 101:327-336

23. Decochez K, de Leeuw IH, Keymeulen B et al (2002) IA-2 autoantibodies predict impending type I diabetes in siblings of patients. Diabetologia 45:1658-1666

24. Achenbach P, Warncke K, Reiter J et al (2004) Stratification of type 1 diabetes risk on the basis of islet autoantibody characteristics. Diabetes 53:384-392

25. Bonifacio E, Bingley PJ, Shattock M et al (1990) Quantification of islet-cell antibodies and prediction of insulin-dependent diabetes. Lancet 335:147-149

26. Achenbach P, Koczwara K, Knopff A, Naserke H, Ziegler AG, Bonifacio E (2004) Mature high-affinity immune responses to (pro)insulin anticipate the autoimmune cascade that leads to type 1 diabetes. J Clin Invest 114:589-597

27. Mayr A, Schlosser M, Grober N et al (2007) GAD autoantibody affinity and epitope specificity identify distinct immunization profiles in children at risk for type 1 diabetes. Diabetes 56:1527-1533

28. Krause S, Chmiel R, Bonifacio E et al (2012) IA-2 autoantibody affinity in children at risk for type 1 diabetes. Clin Immunol 145:224-229

29. Polychronakos C, Li Q (2011) Understanding type 1 diabetes through genetics: advances and prospects. Nat Rev Genet 12:781-792
30. Todd JA (2010) Etiology of type 1 diabetes. Immunity 32:457-467

31. Dendrou CA, Plagnol V, Fung E et al (2009) Cell-specific protein phenotypes for the autoimmune locus IL2RA using a genotypeselectable human bioresource. Nat Genet 41:1011-1015

32. Tang Q, Adams JY, Penaranda C et al (2008) Central role of defective interleukin-2 production in the triggering of islet autoimmune destruction. Immunity 28:687-697

33. Long SA, Cerosaletti K, Bollyky PL et al (2010) Defects in IL-2R signaling contribute to diminished maintenance of FOXP3 expression in $\mathrm{CD} 4(+) \mathrm{CD} 25(+)$ regulatory $\mathrm{T}$ cells of type 1 diabetic subjects. Diabetes 59:407-415

34. Yamanouchi J, Rainbow D, Serra P et al (2007) Interleukin-2 gene variation impairs regulatory $\mathrm{T}$ cell function and causes autoimmunity. Nat Genet 39:329-337

35. Sgouroudis E, Albanese A, Piccirillo CA (2008) Impact of protective IL-2 allelic variants on CD4+ Foxp3+ regulatory T cell function in situ and resistance to autoimmune diabetes in NOD mice. $\mathrm{J}$ Immunol 181:6283-6292

36. Howson JM, Cooper JD, Smyth DJ et al (2012) Evidence of genegene interaction and age-at-diagnosis effects in type 1 diabetes. Diabetes 61:3012-3017

37. Kurreeman FA, Schonkeren JJ, Heijmans BT, Toes RE, Huizinga TW (2004) Transcription of the IL10 gene reveals allele-specific regulation at the mRNA level. Hum Mol Genet 13:1755-1762

38. Smyth DJ, Cooper JD, Bailey R et al (2006) A genome-wide association study of nonsynonymous SNPs identifies a type 1 diabetes locus in the interferon-induced helicase (IFIH1) region. Nat Genet 38:617-619

39. Nejentsev S, Walker N, Riches D, Egholm M, Todd JA (2009) Rare variants of IFIH1, a gene implicated in antiviral responses, protect against type 1 diabetes. Science 324:387-389

40. Smyth DJ, Plagnol V, Walker NM et al (2008) Shared and distinct genetic variants in type 1 diabetes and celiac disease. N Engl J Med 359:2767-2777

41. Vang T, Congia M, Macis MD et al (2005) Autoimmuneassociated lymphoid tyrosine phosphatase is a gain-of-function variant. Nat Genet 37:1317-1319

42. Nejentsev S, Howson JM, Walker NM et al (2007) Localization of type 1 diabetes susceptibility to the MHC class I genes HLA-B and HLA-A. Nature 450:887-892

43. von Herrath M (2009) Diabetes: a virus-gene collaboration. Nature 459:518-519

44. Smyth DJ, Cooper JD, Howson JM et al (2011) FUT2 nonsecretor status links type 1 diabetes susceptibility and resistance to infection. Diabetes 60:3081-3084

45. MacFarlane AJ, Strom A, Scott FW (2009) Epigenetics: deciphering how environmental factors may modify autoimmune type 1 diabetes. Mamm Genome 20:624-632 